\title{
Narrativa
}

\section{Ramona ONNIS e Manuela SPINELLI (a cura di), Donne e Sud. Percorsi nella letteratura italiana contemporanea}

Ada D'Agostino

\section{(2) OpenEdition \\ Journals}

Edizione digitale

URL: https://journals.openedition.org/narrativa/489

DOI: $10.4000 /$ narrativa. 489

ISSN: 2804-1224

Editore

Presses universitaires de Paris Nanterre

\section{Edizione cartacea}

Data di pubblicazione: 1 décembre 2021

Paginazione: $301-303$

ISBN: 978-2-84016-485-2

ISSN: $1166-3243$

Notizia bibliografica digitale

Ada D'Agostino, «Ramona onnis e Manuela spinelu (a cura di), Donne e Sud. Percorsi nella letteratura italiana contemporanea», Narrativa [Online], 43 | 2021, online dal 01 novembre 2021, consultato il 03 novembre 2022. URL: http://journals.openedition.org/narrativa/489 ; DOI: https://doi.org/10.4000/ narrativa.489

Questo documento è stato generato automaticamente il 3 novembre 2022.

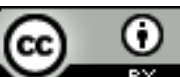

Creative Commons - Attribuzione 4.0 Internazionale - CC BY 4.0 https://creativecommons.org/licenses/by/4.0/ 


\title{
Ramona ONNIS e Manuela SPINELLI (a cura di), Donne e Sud. Percorsi nella letteratura italiana contemporanea
}

\author{
Ada D'Agostino
}

\section{NOTIZIA}

Ramona onNis e Manuela SPINELLI (a cura di), Donne e Sud. Percorsi nella letteratura italiana contemporanea, Firenze, Franco Cesati, 2018, 144 p.

1 Il volume Donne e Sud, si legge nell'Introduzione, intende "riflettere su come le rappresentazioni, gli stereotipi, l'identità stessa delle donne, siano influenzati dall'appartenenza al Sud". La riflessione prende le mosse dalla constatazione che un medesimo ordine di pensiero sembra costringere le donne e i tanti Sud del mondo all'interno di categorie specifiche: entrambi "altro" rispetto a norme falsamente neutrali (l'uomo, il Nord); entrambi definiti da generici "binomi oppositivi"; entrambi, infine, oggetto di una infantilizzazione forzosa e assimilati a vario titolo al mondo naturale (da intendersi come antitetico alle dimensioni della razionalità e della cultura).

2 Il volume si articola in tre sezioni, l'ultima delle quali dedicata alle interviste a due scrittrici: Shirin Ramzanali Fazel e Maria Rosa Cutrufelli. Lo scritto di apertura di Anna Mirabella riporta alla luce un aspetto curiosamente eluso dagli studi di De Martino sul tarantismo in Salento: la componente di genere. Prendendo le mosse dall'altissima frequenza di tarantate donne, Mirabella sottolinea come le interferenze ecclesiastiche sopraggiunte nel rituale possano leggersi come riaffermazione di un potere imposto sull'intera comunità ma prima ancora sul corpo delle donne. Una rilettura del concetto di care orienta lo studio di Angela Biancofiore, che capovolge la relazione tra attività di cura, tradizionalmente assimilata al femminile, e subalternità, sottolineando come il care rappresenti un'esigenza intrinseca a ogni essere umano, e in ragione di ciò 
potrebbe farsi fulcro di "un'etica dell'interdipendenza e dell'alterità"; analizzando le opere di tre scrittrici provenienti da diversi Sud (Wadia, de Caldas Brito e Aquaro), l'autrice stablisice il potere della letteratura di educare all'empatia e alla compassione, nonché all'apertura verso un'alterità. Di capovolgimento della tradizione, e a più livelli, possiamo ancora parlare grazie all'opera di Jolanda Insana, qui ripercorsa dal saggio di Emanuele Broccio. La parola poetica insaniana, profondamente radicata alla Sicilia, riporta perentoriamente al centro della scena la donna attraverso un'operazione di riappropriazione di quegli stessi spazi negati alle donne, siano essi legati alla vita reale o alla vita letteraria. Chiude la sezione il saggio di Ramona Onnis sulla saga dei Chironi, trilogia di Marcello Fois che ripercorre la storia di tre generazioni di famiglie sarde. La trilogia stempera le differenze Nord/Sud; e soprattutto, mette in scena donne "determinate e complete", capaci di riaffermarsi a prescindere da qualunque appartenenza geografica.

Un approdo simile sembra orientare lo studio di Silvia Contarini che apre la seconda sezione, e si concentra sulle opere di due grandi autrici del Novecento: Una donna di Sibilla Aleramo e L'arte della gioia di Goliarda Sapienza. Un dato comune emerge dalle analisi di Contarini: l'emancipazione della donna è un percorso interiore, privato, una consapevolezza profonda che può raggiungersi a prescindere dai luoghi che si abitano; la prevaricazione maschile investe e attraversa, in maniera indifferenziata, ogni ceto sociale e ogni latitudine. È ancora un percorso accidentato ma segnato da una forte determinazione quello descritto da Daniele Comberiati, che analizza la figura della brigantessa nei romanzi La briganta e Coccarde rosse, rispettivamente di Maria Rosa Cutrufelli e Annalisa Bari. Nelle opere si intrecciano due necessità: l'autodeterminarsi della donna, che scegliendo attivamente forgia e raggiunge la sua identità; e la necessità di recuperare quelle voci dissonanti rispetto a una storia univocamente imposta. Dalla risignificazione storica passiamo, col saggio di Serena Todesco, alla risemantizzazione spaziale, esaminata attraverso l'opera e l'esperienza Elvira Mancuso, Maria Occhipinti e Gisella Modica. La prima farà della casa "un luogo di pratica politica, sovvertendone la natura di spazio chiuso perché sessuato", finendo dunque col riabi(li)tare lo spazio domestico attraverso un sovvertimento di significato. La seconda occupa col suo corpo - gravido e quindi, in un certo senso, perentoriamente connotato - lo spazio della strada; spazio che diventa plurale - l'intrico delle strade di Palermo nell'opera di Modica, la cui protagonista rifiuta lo spazio chiuso e logoro della casa per scontrarsi con la realtà labirintica e polifonica del capoluogo siculo. Il saggio di Nadia Setti ripercorre infine la relazione con lo spazio meridiano attraverso le esperienze antitetiche di due donne, protagoniste della saga L'amica geniale di Elena Ferrante: una, Elena (Lenù), dislocata, l'altra, Raffaella (Lila), rimasta al centro della realtà napoletana. Lo spazio - oltre lo spazio dell'idioma materno, del corpo della madre veicolato da parole intraducibili: frantumaglia - diventa mobile e in fondo non determinante: per dirla con Setti, "la risposta alla domanda sulla provenienza/appartenenza si potrebbe [...] scomporre in vari verbi di movimento" (p. 124).

4 Le interviste, infine, danno la parola a due autrici che rimettono al centro dei loro racconti le donne: appartenenti a diverse generazioni, spesso vittime di pregiudizi razziali e di classe, o di una storia che ha tolto loro la voce, incrinano il modello patriarcale che vuole il femminile passivo e soggiogato. Mostrando almeno in parte la possibilità di una rappresentazione che dia conto dell'esistenza di una pluralità di 
individue, non dissimile, in fondo, da quella pluralità di Sud (e di Nord) che non accetta definizioni univoche. 